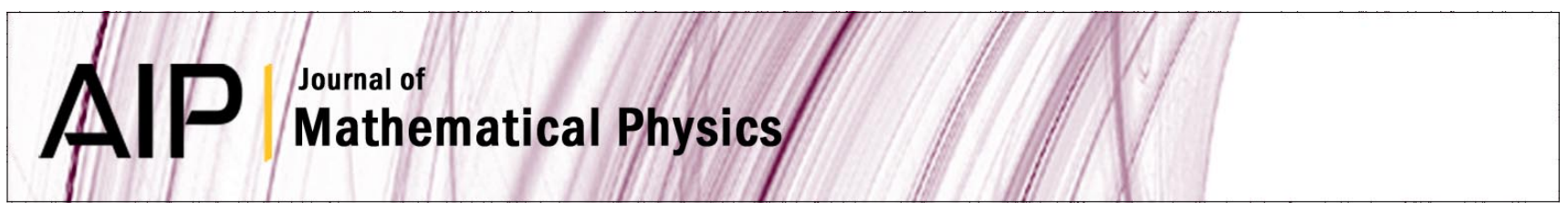

\title{
Inverse scattering problems: A study in terms of the zeros of entire functions
}

M. NietoVesperinas

Citation: J. Math. Phys. 25, 2109 (1984); doi: 10.1063/1.526369

View online: http://dx.doi.org/10.1063/1.526369

View Table of Contents: http://jmp.aip.org/resource/1/JMAPAQ/v25/i6

Published by the American Institute of Physics.

\section{Related Articles}

Using scattering theory to compute invariant manifolds and numerical results for the laser-driven Hénon-Heiles system

Chaos 22, 043138 (2012)

Practical axial optical trapping

Rev. Sci. Instrum. 83, 103106 (2012)

Multiangle static and dynamic light scattering in the intermediate scattering angle range

Rev. Sci. Instrum. 83, 093106 (2012)

Modulated heterodyne light scattering set-up for measuring long relaxation time at small and wide angle Rev. Sci. Instrum. 83, 083102 (2012)

An in-vacuum $x$-ray diffraction microscope for use in the $0.7-2.9 \mathrm{keV}$ range

Rev. Sci. Instrum. 83, 033703 (2012)

\section{Additional information on J. Math. Phys.}

Journal Homepage: http://jmp.aip.org/

Journal Information: http://jmp.aip.org/about/about_the_journal

Top downloads: http://jmp.aip.org/features/most_downloaded

Information for Authors: http://jmp.aip.org/authors

\section{ADVERTISEMENT}

The most comprehensive support for physics in any mathematical software package World-leading tools for performing calculations in theoretical physics

www.maplesoft.com/physics
Your work in Maple matches how you would write the problems and solutions by hand

- State-of-the-art environment for algebraic computations in physics

The only system with the ability to handle a wide range of physics computations as well as pencil-and-paper style input and textbook-quality display of results

- Access to Maple's full mathematical power, programming language, visualization routines, and document creation tools 


\title{
Inverse scattering problems: A study in terms of the zeros of entire functions
}

\author{
M. Nieto-Vesperinas ${ }^{a)}$ \\ Instituto de Optica del C.S.I.C., Serrano 121, Madrid-6, Spain
}

(Received 30 March 1983; accepted for publication 14 October 1983)

\begin{abstract}
The study of the zeros of functions of several complex variables recently reported is applied to scalar scattered wavefields. First, an analysis of the role of the zeros of the scattering amplitude leads to an interpretation of the nonuniqueness of inverse source and scattering problems.

Secondly, the analytic character of the scattered wavefunction outside the scattering volume and its zero description, as well as its role for information transmission, is discussed.
\end{abstract}

PACS numbers: 42.10.Hc, 03.65.Sq, 02.30.Dk, 02.30.Jr

\section{INTRODUCTION}

The analyticity and unique continuation of solutions to partial differential equations has been discussed in several contexts in physics (see, e.g., Ref. 1). One of the applications has been, among others, the description of scalar scattered wavefields in the far zone, i.e., when the scattered field $u^{(s)}(r)$, $(\mathbf{r}=(\mathrm{x}, \mathrm{y}, \mathrm{z}))$ and the spectral amplitude $A\left(k_{0} \mathbf{s}_{\perp}\right)$,

$\left[k_{0} s=k_{0}\left(s_{1}, s_{2}, s_{3}\right), k_{0}=2 \pi / \lambda\right.$, and $s$ being a unit vector in the direction of observation. $k_{0} \mathrm{~s}_{1}=k_{0}\left(s_{1}, s_{2}\right)$ is the projection of the 3-D wavevector $k_{0}$ s onto the $X Y$-plane so that $\left|\mathbf{s}_{1}\right|^{2}+s_{3}^{2}$ $=1, s_{3}=+{\sqrt{1-s_{1}}}^{2}$ and $\left.\left|k_{0} \mathbf{s}_{1}\right| \leqslant\left|k_{0} \mathbf{s}\right|=k_{0}\right]$ are connected by the asymptotic expression ${ }^{2}$

$$
u^{(s)}(\mathbf{r})=-2 \pi i k_{0} s_{3} A\left(k_{0} \mathbf{s}_{1}\right) \frac{e^{i k_{0} r}}{r} .
$$

So far these discussions have been first restricted to functions $A(s)$ of one scalar variable $s(\operatorname{Refs} .3-5)$ and, within this limitation, they have addressed the ambiguity in object reconstruction from far-zone intensity data (phase problem) and put forward methods of solution based on the localization of the zeros of $A(s)$ in the complex plane $\hat{s}=s+i s^{\prime} .^{5-8}$ Analytic Fourier Optics, as this analysis of $A(s)$ was coined, ${ }^{9}$ also provides a means of encoding information, which is al ternative to the Shannon description of sampling and interpolation of bandlimited functions. Recently, some generalizations of the study of the above ambiguity have been done by means of the theory of entire functions of two and more complex variables. ${ }^{10-12}$

The purpose of this paper is twofold. We shall see that the angular spectrum $A\left(K_{1}, K_{2}\right)$ is the restriction of the 3-D Fourier transform $F\left(K_{1}, K_{2}, K_{3}\right)$ of the source function to values of $K_{3}=q$ satisfying $|\mathbf{K}|^{2}+q^{2}=k_{0}^{2}, \mathbf{K}=\left(K_{1}, K_{2}\right)$, for any real value of $|\mathbf{K}|$. Thus, based on the theory of Fourier transforms in several complex variables we shall discuss first in Sec. 2 how the study of the analytic continuation of $F$ into three complex variables allows an interpretation of the nonuniqueness of the inverse source and scattering problems, ${ }^{13,14}$ within the framework of the theory of entire functions. Specifically, we shall base our discussion on the recent paper by Manolitsakis ${ }^{12}$ on two-dimensional scattered fields, which are given by a Fourier transform, considered as functions of two-complex variables. As such, we shall use its

\footnotetext{
a) Temporary address: The Institute of Optics, The University of Rochester, Rochester, New York 14627.
}

description in terms of "zero lines" of a function of several complex variables [where a "zero line" in the $n$-dimensional complex space means an $(n-1)$-dimensional surface in which the function vanishes]. We shall not present in detail these mathematical essentials as they are presented in depth in Ref. 12. We will instead concentrate on some consequences extracted from that theory. In particular, a description of the source Fourier transforms $F\left(\widehat{K}_{1}, \widehat{K}_{2}, \widehat{K}_{3}\right)$ in terms of the zero lines of its analytic continuation into the 6-D complex space $\left(\widehat{K}_{1}, \widehat{K}_{2}, \widehat{K}_{3}\right)$ justifies, by virtue of a theorem due to Osgood, the existence of identically zero scattering amplitudes given as data of a single or a discrete number of scattering experiments. ${ }^{14.15}$

Secondly, in Sec. 3 we shall address the study of the analyticity of the scattered field without the approximations introduced for studying its far-zone behavior. We shall see that between this region and the scattering object, and more specifically, outside the scattering volume and its boundary, the field may be described in its free propagation by an analytic function of the spatial complex variables $(\hat{x}, \hat{y}, \hat{z})$. The kind of analyticity may be even more specified in those regions in which the scattered field admits a representation in terms of the angular spectrum of plane waves. As a matter of fact, given a certain level of noise this field may be approximated at optical frequencies by an entire function of exponential type at those points where evanescent waves from a

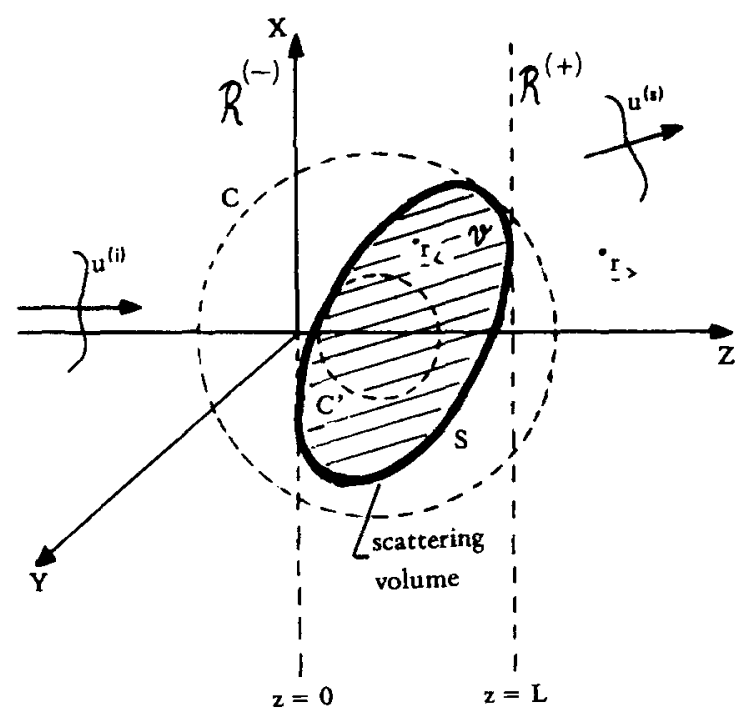

FIG. 1. Scattering geometry. 
certain spatial frequency $K=\sqrt{K_{1}^{2}+K_{2}^{2}}>k_{0}$ onwards are not detected and thus may be neglected. The characterization of this scattered field by "zero lines" is a difficult one in several complex variables. ${ }^{12}$ However, in those problems exhibiting cylindrical symmetry, the scattered field may be described at each distance $z$ from the object by an entire function of exponential type $u^{(s)}(\hat{x})$ of a single variable; as such, its propagation may be described by means of its zero trajectories giving a zero distribution with a certain density at each complex plane associated with a given distance $z$, which decreases, as this distance increases, up to the limit marked by the evanescent waves. The reconstruction of the field in the process reversal to that of propagation from the object poses, therefore, the problem of reproducing higher zero densities as one gets closer to the scattering volume.

\section{ANALYTIC PROPERTIES OF THE 3-D SOURCE FOURIER TRANSFORM}

Let $u(\mathbf{r})$ represent a scalar monochromatic wavefield in the presence of a scattering medium that occupies a finite volume $v$ in the strip $0<z<L$ (Fig. 1). $u(\mathbf{r})$ satisfies at any point $\mathbf{r}=(x, y, z)$ of the space the inhomogeneous Helmholtz equation

$$
\left(\nabla^{2}+k_{0}^{2}\right) u(\mathbf{t})=V(\mathbf{r}) u(\mathbf{r})
$$

where the scattering potential is given in terms of the refractive index $n(\mathbf{r})$ of the scattering medium by

$$
\begin{array}{lll}
V(\mathbf{r})=-k_{0}^{2}\left[n^{2}(\mathbf{r})-1\right], & & \mathbf{r} \in v, \\
V(\mathbf{r})=0, & & \mathbf{r} \notin v,
\end{array}
$$

$k_{0}=2 \pi / \lambda$ being the wavenumber in free space.

In order to write the integral equations describing the propagation of the field $u(\mathbf{r})$ we shall follow a procedure already employed by Wolf. ${ }^{15}$

Let $\mathbf{r}_{>}$and $\mathbf{r}_{<}$denote respectively the position vectors of a point exterior and interior to the scattering volume $v$, then the application of Green's theorem yields for the exterior field ${ }^{16}$ :

(i) In terms of the field $u\left(\mathbf{r}^{\prime}\right)$ inside the volume $v$ :

$$
u\left(\mathbf{r}_{>}\right)=u^{(i)}\left(\mathbf{r}_{>}\right)-\frac{1}{4 \pi} \int_{v} d^{3} \mathbf{r}^{\prime} G\left(\mathbf{r}_{>}, \mathbf{r}^{\prime}\right) V\left(\mathbf{r}^{\prime}\right) u\left(\mathbf{r}^{\prime}\right) .
$$

(ii) As a function of the values of the field and its normal derivative on the surface $S$ which constitutes the boundary of $v$ :

$$
\begin{aligned}
u\left(\mathbf{r}_{>}\right)= & u^{(i)}\left(\mathbf{r}_{>}\right)+\frac{1}{4 \pi} \int_{S} d s\left[u\left(\mathbf{r}^{\prime}\right) \frac{\partial G\left(\mathbf{r}_{>}, \mathbf{r}^{\prime}\right)}{\partial n}\right. \\
& \left.-G\left(\mathbf{r}_{>}, \mathbf{r}^{\prime}\right) \frac{\partial u\left(\mathbf{r}^{\prime}\right)}{\partial n}\right] .
\end{aligned}
$$

In Eqs. (4) and (5) $u^{(i)}(\mathbf{r})$ denotes the incident field, and the integral of the second term represents the scattered field $u^{(s)}(\mathbf{r}) . G\left(\mathbf{r}, \mathbf{r}^{\prime}\right)$ is the outgoing free space Green's function:

$$
G\left(\mathbf{r}, \mathbf{r}^{\prime}\right)=\frac{e^{i k_{0}\left|\mathbf{r}-\mathbf{r}^{\prime}\right|}}{\left|\mathbf{r}-\mathbf{r}^{\prime}\right|},
$$

which is the solution of the equation

$$
\left(\nabla^{2}+k_{0}^{2}\right) G\left(\mathbf{r}, \mathbf{r}^{\prime}\right)=-4 \pi \delta\left(\mathbf{r}-\mathbf{r}^{\prime}\right) .
$$

$G\left(\mathbf{r}, \mathbf{r}^{\prime}\right)$ admits the decomposition (Weyl) ${ }^{15}$

$$
G\left(\mathbf{r}, \mathbf{r}^{\prime}\right)=\frac{i}{2 \pi} \iint_{-\infty}^{\infty} \frac{d^{2} \mathbf{K}}{q} e^{i\left[\mathbf{K} \cdot\left(\mathbf{R}-\mathbf{R}^{\prime}\right)+q\left|z-z^{\prime}\right|\right]},
$$

where $\mathbf{r}=(\mathbf{R}, z)$ and $\mathbf{r}^{\prime}=\left(\mathbf{R}^{\prime}, z^{\prime}\right)\left(\mathbf{R}\right.$ and $\mathbf{R}^{\prime}$ being vectors in a plane perpendicular to $0 \mathrm{Z}$ ). Also

$$
\begin{aligned}
& q=+\sqrt{k_{0}^{2}-K^{2}} \text { when }|\mathbf{K}| \leqslant k_{0}, \\
& q=+i \sqrt{K^{2}-k_{0}^{2}} \quad \text { when }|\mathbf{K}|>k_{0} .
\end{aligned}
$$

Denoting by $R^{(+)}$and $R^{(-)}$, respectively, the half-spaces $z<0$ and $z>L$ (Fig. 1), the absolute value $\left|z-z^{\prime}\right|$ may be written as

$$
\begin{aligned}
& \left|z-z^{\prime}\right|=+\left(z-z^{\prime}\right), \text { when } \mathbf{r} \in R^{(+)} \text {and } \mathbf{r}^{\prime} \in v+S, \\
& \left|z-z^{\prime}\right|=-\left(z-z^{\prime}\right) \text {, when } \mathbf{r} \in R^{(-)} \text {and } \mathbf{r}^{\prime} \in v \text {. }
\end{aligned}
$$

By substituting Eq. (8) into Eqs. (4) and (5), making use of Eqs. (10) and changing the order of integration, we obtain for the scattered field ${ }^{17}$ :

$$
u^{(s)}\left(\mathbf{r}_{>}\right)=\iint_{-\infty}^{\infty} d^{2} \mathbf{K} A^{ \pm}(\mathbf{K}) e^{\left.i \mid \mathbf{K} \cdot \mathbf{R}> \pm q^{2},\right)},
$$

where the angular spectrum, or scattering amplitude, $A^{( \pm)}(\mathbf{K})$ may be written either as a volume integral [cf. Eq. (4)]

$$
A^{( \pm)}(\mathbf{K})=\frac{-i}{8 \pi^{2}} \frac{1}{q} \int_{v} d^{3} \mathbf{r} V(\mathbf{r}) u\left(\mathbf{r}^{\prime}\right) e^{-i\left(\mathbf{K} \cdot \mathbf{R}^{\prime} \pm q z^{\prime}\right)}
$$

or as a surface integral [cf. Eq. (5)]

$$
\begin{aligned}
A^{( \pm \prime}(\mathbf{K})= & \frac{i}{8 \pi^{2}} \frac{1}{q} \int_{S} d s\left[u\left(\mathbf{r}^{\prime}\right) \frac{\partial}{\partial n}\left(e^{-i\left(\mathbf{K} \cdot \mathbf{R}^{\prime} \pm q z^{\prime}\right)}\right)\right. \\
& \left.-e^{-i\left(\mathbf{K} \cdot \mathbf{R}^{\prime} \pm q z^{\prime}\right)} \frac{\partial u}{\partial n}\right] .
\end{aligned}
$$

But one has

$\frac{\partial}{\partial n}\left[e^{\left.-i \mid \mathbf{K} \cdot \mathbf{R}^{\prime} \pm q z^{\prime}\right)}\right]=-i \mathbf{n} \cdot(\mathbf{K}, \pm q) e^{-i\left(\mathbf{K} \cdot \mathbf{R}^{\prime} \pm q z^{\prime}\right)}$

That is

$$
\begin{aligned}
A^{( \pm)}(\mathbf{K})= & \frac{-i}{8 \pi^{2} q} \int_{S} d s\left[i \mathbf{n} \cdot(\mathbf{K}, \pm q) u\left(\mathbf{r}^{\prime}\right)\right. \\
& \left.+\frac{\partial u\left(\mathbf{r}^{\prime}\right)}{\partial n}\right] e^{-i\left(\mathbf{K} \cdot \mathbf{R}^{\prime} \pm q z^{\prime}\right)}
\end{aligned}
$$

which reduces to the usual expression for the angular spectrum in terms of $u\left(\mathbf{R}^{\prime}, L\right)$ when the surface $S$ is taken as the plane $z=L$ [cf. Eq. (2.4) of Ref. 18] and one chooses Sommerfeld's Green's function. ${ }^{19}$

The upper $(+)$ and lower $(-)$ signs in Eqs. (12)-(15) are taken according to whether the point $\mathbf{r}_{>}$belongs either to $R^{(+)}$or $R^{(-)}$.

As is well known, those components of the scattered field, Eq. (1), for which Eq. (9a) holds, represent homogeneous waves and those components satisfying Eq. (9b) are evanescent waves, whose contribution may be significant at points $\mathbf{r}_{>}$near the boundary $S$; and certainly, if the dimensions of the scattering volume are of the order of, or smaller than, the wavelength $\lambda$ of the incident field (or if the scale of inhomogeneity of the scattering medium is of the order of, or smaller than $\lambda$ ), then, the contribution of the evanescent waves in the expression (11) may be important. ${ }^{20-22}$ 
Considering the representation (12) for $A^{( \pm)}(\mathbf{K})$, we shall introduce

$$
f\left(\mathbf{r}^{\prime}\right)=V\left(\mathbf{r}^{\prime}\right) u\left(\mathbf{r}^{\prime}\right)
$$

which is sometimes called the object wave or source function $^{4,13}$ and it is such that, due to Eqs. (3), becomes zero outside the volume $v$; i.e., $f\left(\mathbf{r}^{\prime}\right)$ is a function of finite support $v$. As a consequence, its three-dimensional Fourier transform $F(\mathbf{k})$ :

$$
\begin{aligned}
& F(\mathbf{k})=\frac{1}{(2 \pi)^{3}} \int_{-\infty}^{\infty} d^{3} \mathbf{r}^{\prime} f\left(\mathbf{r}^{\prime}\right) e^{-\mathbf{k} \cdot \mathbf{r}^{\prime}}, \\
& \mathbf{k}=\left(K_{1}, K_{2}, K_{3}\right)
\end{aligned}
$$

is, by virtue of the Plancherel-Polya theorem, ${ }^{23}$ the boundary value for real values of $\mathbf{k}$ of an entire function of exponential type in the $\hat{\mathbf{k}}$ complex space of the three complex variables $\hat{\mathbf{k}}=\left(\widehat{\boldsymbol{K}}_{1}, \widehat{\boldsymbol{K}}_{2}, \widehat{\boldsymbol{K}}_{3}\right)\left(\widehat{K}_{1}=K_{1}+i K_{1}^{\prime}, \widehat{K}_{z}=K_{2}+i K_{2}^{\prime}\right.$, $\left.\widehat{K}_{3}=K_{3}+i K_{3}^{\prime}\right)$, i.e., such that $F(\hat{\mathbf{k}})$ always satisfies the inequality

$$
\left|F\left(\widehat{\boldsymbol{K}}_{1}, \widehat{\boldsymbol{K}}_{2}, \widehat{\boldsymbol{K}}_{3}\right)\right| \leqslant C e^{a\left(\left|\hat{\boldsymbol{K}}_{1}\right|+\left|\widehat{K}_{2}\right|+\left|\hat{\boldsymbol{K}}_{3}\right|\right.},
$$

where $C$ and $a$ are positive constants.

Comparing Eqs. (11) and (16) we obtain

$$
\begin{aligned}
& A^{( \pm)}(\mathbf{K})=\frac{\pi}{i q} F\left(\mathbf{k}^{( \pm \eta}\right), \\
& \mathbf{k}^{( \pm)}=\left(K_{1}, K_{2}, \pm q\right),
\end{aligned}
$$

i.e., $E q$. (19) allows us to express the angular spectrum $A^{\prime \pm}(\mathbf{k})$ in terms of the 3-D Fourier transform $F(\mathbf{k})$ of the source function restricted to the conditions (9). It has been already noted ${ }^{24}$ that for values $|\mathbf{k}|>K_{0}, q$ is imaginary and $A^{( \pm)}(\mathbf{k})$ represents the value of $F(\hat{\mathbf{k}})$ along the "line" given by Eq. (9b).

So far only real values of $\mathbf{K}$ have been considered in the path of integration corresponding to Eq. (8). If we make a transformation to cylindrical coordinates

$$
K_{1}=\zeta \cos \alpha, \quad K_{2}=\zeta \sin \alpha,
$$

then Eq. (7) may be written

$$
G\left(\mathbf{r}, \mathbf{r}^{\prime}\right)=i \int_{0}^{\infty} \frac{d \zeta \zeta}{\sqrt{k_{0}^{2}-\zeta^{2}}} J_{0}\left(\zeta\left|\mathbf{R}-\mathbf{R}^{\prime}\right|\right) e^{i \sqrt{k_{0}^{2}-\zeta^{2}}\left|z-z^{\prime}\right|} .
$$

The above integral may be extended to negative values of $\zeta$ by expressing the zero order Bessel function $J_{0}$ in terms of the Hankel function of the first kind and order zero $H_{0}^{1}$

$$
J_{0}(z)=\frac{1}{2}\left[H_{0}^{1}(z)-H_{0}^{1}(-z)\right]
$$

so that

$$
G\left(\mathbf{r}, \mathbf{r}^{\prime}\right)=\frac{i}{2} \int_{-\infty}^{\infty} \frac{d \zeta \zeta}{\sqrt{k_{0}^{2}-\zeta^{2}}} H_{0}^{1}\left(\zeta\left|\mathbf{r}-\mathbf{r}^{\prime}\right|\right) e^{i \sqrt{k_{0}^{2}-\zeta^{2}} \mid z-z^{\prime}} .
$$

The path of integration in $(20 \mathrm{~b})$ may be modified in the complex $\xi$-plane by choosing any other path with ends $(-\infty, \infty)$ providing one avoids to cross the cuts $\xi \equiv \operatorname{Re}$ $\{\hat{\zeta}\}=\operatorname{Re}\left\{\lambda k_{0}\right\}, \zeta^{\prime} \equiv \operatorname{Im}\{\hat{\zeta}\{\gtrless 0$ arising from the two branch points at $\zeta= \pm k_{0}$. The choice of such a path with complex values $\hat{\xi}$ would imply a decomposition of the exterior scattered field $u^{(s)}\left(\mathbf{r}_{>}\right)$, Eq. (4), into inhomogeneous waves, alter- native to the representation (11) in terms of homogeneous and evanescent components.

Following now the analysis of Ref. 12 and returning to Eq. (17), a central result of the theory of functions of several complex variables is that a function $F\left(\widehat{K}_{1}, \widehat{K}_{2}, \widehat{K}_{3}\right)$ that is an entire function of exponential type in the $\hat{\mathbf{k}}$-space has no isolated zero points ${ }^{23}$; i.e., their zeros are always continuous lines [here "line"means of course a 4-dimensional variety of the 6-D complex $\hat{\mathbf{k}}$-space given by a functional dependence between the three complex variables $\left.\left(\widehat{K}_{1}, \widehat{K}_{2}, \widehat{K}_{3}\right)\right]$. An entire function of exponential type has an infinite number of zero lines. ${ }^{12}$

The set of zeros of $F\left(\widehat{K}_{1}, \widehat{K}_{2}, \hat{K}_{3}\right)$ is called a reducible analytic set if it can be decomposed into a union of subsets. The entire function $F\left(\widehat{K}_{1}, \widehat{K}_{2}, \widehat{K}_{3}\right)$ is called globally reducible if it may be written as the product of two entire functions. Otherwise it is called globally irreducible. (Global irreducibility does not imply that $F$ is irreducible everywhere.)

The main fundamental theorem that is required in order to characterize an entire function of several complex variables is the following due to Osgood (see a proof in Ref. 25):

Theorem: An entire function $F\left(\widehat{K}_{1}, \widehat{K}_{2}, \widehat{K}_{3}\right)$ that has zeros and does not vanish identically can be 1 niquely decomposed into a finite or infinite product of globalı. irreducible factors in the form:

$F\left(\widehat{K}_{1}, \widehat{K}_{2}, \widehat{K}_{3}\right)=\prod_{m=1}^{N}\left[F_{m}(\hat{\mathbf{k}}) e^{\gamma_{m}(\hat{\mathbf{k}})}\right]^{I_{m}}$,

where $F_{m}(\hat{\mathbf{k}})$ are globally irreducible entire functions, exp $\left[\gamma_{m}(\hat{\mathbf{k}})\right]$ are convergence factors with $\gamma_{m}(\hat{\mathbf{k}})$ being polynomials in $\mathbf{k}$ and the exponents $l_{m}$ are integers.

Let us compare the decomposition given by Eq.(21) with that corresponding to an entire function of one complex variable $\widehat{K}^{5}$ :

$$
F(\hat{K})=C \widehat{K}^{\mu} e^{\alpha \hat{K}} \prod_{n=-\infty}^{\infty}\left(1-\frac{\widehat{K}}{\widehat{K}_{n}}\right) e^{\widehat{K} / \widehat{K}_{n}},
$$

where $C, \mu$, and $\alpha$ are constants and $\widehat{K}_{n}$ are the zero points of $F(\hat{K})$.

The main difference between (21) and (22) is that each factor $F_{m}(\hat{\mathbf{k}}) e^{\gamma_{m}(\hat{K})}$ is not known and depends entirely on the zero set of $F(\hat{\mathbf{k}})$. Also, each factor of Eq. (21) may contain not only one, but a certain number (finite or infinite) of zero lines with their possible branches or pseudobranches that cannot be separated. ${ }^{12}$ While in the case of functions of a single complex variable all the information about the function is contained in the position of its zeros $\widehat{K}_{n}$. For functions of several complex variables, however, it is necessary to know not only the position but also the relationship between zero lines and its combination in each irreducible factor. The uniqueness of irreducible zero sets ensures that each irreducible factor determines in an unique manner the function $F\left(\widehat{K}_{1}, \widehat{K}_{2}, \widehat{K}_{3}\right)$. The uniqueness of the product ensures that each function $F\left(\widehat{K}_{1}, \widehat{K}_{2}, \widehat{K}_{3}\right)$ is uniquely determined by the zero lines.

The asymptotic behavior of $F\left(\widehat{K}_{1}, \widehat{K}_{2}, \widehat{K}_{3}\right)$ is defined by the shape of the support of $f(\mathbf{r})$,i.e., by the shape of the volume $v$. For example, a function $f(\mathbf{r})$ contained in a spherical volume will yield a 3-D Fourier transform that behaves asymp- 
totically as $\left(1 / \hat{k}^{2}\right)[\sin a \hat{k}-a \hat{k} \cos a \hat{k}]$,

$\hat{k}=\sqrt{K_{1}^{2}+K_{2}^{2}+K_{3}^{2}}$ which is the 3-D Fourier transform of a sphere of radius $a$. Its zeros are given by the lines $\hat{k}=\hat{k}_{0}$, where $\hat{k}_{0}$ are the values that are solutions of the equation $\tan a \hat{k}=a \hat{k}$.

Th existence of an infinity of zero lines of an entire function of exponential type $F\left(\widehat{K}_{1}, \widehat{K}_{2}, \hat{K}_{3}\right)$ allows an interpretation of nonuniqueness of object wave reconstruction from the value of $A^{( \pm)}(\mathbf{K})$ obtained from a single experiment. ${ }^{14}$ In fact, there may exist an object wave $f_{0}(\mathbf{r})$ such that, for a given incident field, its 3-D Fourier transform $F\left(\widehat{K}_{1}, \widehat{K}_{2}, \widehat{K}_{3}\right)$ given by Eq. (16) has one of its zero lines given by Eq. (8), i.e., such that $A_{0}{ }^{ \pm}(\mathbf{k})$ is zero. Then, due to Eq. (11) the corresponding scattered field $u_{0}^{(s)}\left(\mathbf{r}_{>}\right)$would be zero in $R^{(+)}$or $R^{(-)}$. Also since the object wave, Eq. (16), is a function localized within $v$ then the angular spectrum

$$
A^{\prime \prime \pm}(\mathbf{K})=A_{0}^{( \pm \prime}(\mathbf{K})+A^{\prime \pm \prime}(\mathbf{K})
$$

would be equal to $A^{( \pm)}(\mathrm{K})$ and thus the corresponding scattered field

$$
u^{\prime(s)}\left(\mathbf{r}_{>}\right)=u_{0}^{(s)}\left(\mathbf{r}_{>}\right)+u^{(s)}\left(\mathbf{r}_{>}\right)
$$

would be equal to $u^{(s)}\left(\mathbf{r}_{>}\right)$in $R^{(+)}$or $R^{(-)}$.

Moreover, as was noted in Ref. 19, from Eqs. (12) and

(23) it is evident that within $v$ one has

$$
V^{\prime}(\mathbf{r})=\frac{V(\mathbf{r}) u(r)+f_{0}(\mathbf{r})}{u(\mathbf{r})+u_{0}(\mathbf{r})} .
$$

For a given incident field $u^{(i)}(\mathbf{r})=\exp \left(i \mathbf{k}^{(i)} \cdot \mathbf{r}\right)$, $\mathbf{k}^{(i)}=\left(K_{1}^{(i)}, K_{2}^{(i)}, K_{3}^{(i)}\right), k_{0}^{2}=K_{1}^{(i)^{2}}+K_{2}^{(i)^{2}}+q^{(i)^{2}}$, and within the domain of validity of the first Born approximation the angular spectrum $A_{B}^{( \pm)}(\chi)$ is given by

$$
A_{B}^{( \pm)}(\chi)=\frac{\pi}{i q} F\left(\mathbf{x}^{( \pm)}\right)
$$

with the transfer vector

$\mathbf{x}^{( \pm)}=\left(\chi, x_{3}^{( \pm)}\right)=\left(K_{1}-K_{1}^{(i)}, K_{2}-K_{2}^{(i)}, \pm q-q^{(i)}\right)$ lying on the surface

$$
\mathbf{x}^{( \pm 1} \cdot \mathbf{x}^{( \pm)}=2 k_{0}^{2}-2 \mathbf{k}^{( \pm)} \cdot \mathbf{k}^{(i)} \text {. }
$$

For a discrete number of incident wave vectors $\mathbf{k}^{(i)}$, one may have a discrete number of lines (27) being zeros of $F\left(\hat{\mathbf{x}}^{( \pm)}\right)$and, thus, allowing to express $F\left(\hat{\mathbf{x}}^{( \pm)}\right)$by means of a product of irreducible factors of the form of Eq. (21). Thus, as it was already noted ${ }^{14}$ it follows as above that a discrete number of experiments, each with an incident plane wave of wave vector $\mathbf{k}^{(i)}$ does not allow, within the first Born approximation, to uniquely determine the object wave from the angular spectrum $A_{B}^{( \pm)}(\mathrm{X})$.

\section{ANALYTICITY OF THE SCATTERED FIELD}

In order to assess the analytic characteristics of the scattered field $u^{(s)}(\mathbf{r})$, we shall recall the Cauchy-Kowalewski theorem of the theory of partial differential equations (see Ref. 1 for a proof and detailed discussion):

Theorem: There is exactly one analytic solution of the partial differential equation

$$
\nabla^{2} u(x, z)=g\left(x, z, u, \frac{\partial u}{\partial x}, \frac{\partial u}{\partial z}\right)
$$

that satisfies the Cauchy boundary conditions on a smooth boundary $C$ of a bounded simply connected domain $D$ : $\phi=\left.u(x, z)\right|_{C}, \psi=\partial u(x, z) /\left.\partial n\right|_{C}, g$ being an analytic function of its five arguments and $u(x, z)$ being twice continuously differentiable in a real neighborhood of $x=0, z=0$.

Besides (see Refs. 1, 26, and 27), $u(x, z)$ may be analytically continued to complex values $\hat{x}=x+i x^{\prime}, \hat{z}=z+i z^{\prime}$, so that $u(\hat{x}, \hat{z})$ satisfies the Cauchy-Riemann equations with respect to each complex variable, and reduces for $x^{\prime}=z^{\prime}=0$ to the function $u(x, z)$.

These results established for two variables $x, z$ may be also obtained for more independent variables (cf. Ref. 1 and references therein).

Equation (1) does not satisfy the conditions of the above theorem since the source function $f(r)$ is discontinuous across the boundary $S$. However the Helmholtz equation holds the above conditions in the domain exterior to the scattering volume $v$ and its boundary $S$ where $f\left(\mathbf{r}_{>}\right) \equiv 0$. This implies that $u^{(s)}(\mathbf{r})$ fails to be analytic inside the volume $v$ or on its boundary $S$. In fact the scattered field $u^{(s)}(\mathbf{r})$ must have singularities associated to the scattering sources somewhere in $v$ or on its boundary $S$ since if it were analytic everywhere it would be identically zero. ${ }^{26}$ Wave functions analytic in the whole space are the incident field $u^{(i)}(\mathbf{r})$ and any other sourcefree field that satisfies the equation $\left(\nabla^{2}+k_{0}^{2}\right) u(\mathbf{r})=0$ everywhere. ${ }^{28}$ Also, from the representations (4) or (5) for $u\left(\mathbf{r}_{>}\right)$ one can see that since $G\left(\mathbf{r}_{>}, \mathbf{r}^{\prime}\right)$ depends analytically on $\mathbf{r}$, and $\mathbf{r}^{\prime}$ then $\boldsymbol{u}^{(s)}\left(\mathbf{r}_{>}\right)$is an analytic function $\mathbf{r}>$ provided that $\left|\mathbf{r}_{>}-\mathbf{r}^{\prime}\right| \neq 0$ and it may be analytically continued to complex values $\left(\hat{x}_{>}, \hat{y}_{>}, \hat{z}_{>}\right) \cdot{ }^{27}$ According to Refs. 28 and 30 a sufficient condition for this is that $r_{>}$be outside the circumscribed sphere $C$ to $S$ (Fig. 1). ${ }^{30}$ This is certainly true when $\mathbf{r}_{\text {> }}$ is either in $R^{(-)}$or $R^{(+)}$which is also when the representation (10) by means of the angular spectrum converges. ${ }^{30}$ If moreover, the boundary $S$ is analytic, then the values $u^{(s)}\left(\mathbf{r}_{>}\right)$ may be analytically continued up to $S$ (Refs. 29 and 31); this is also a necessary, although not sufficient, condition, for the validity of the angular spectrum representation (1) between $S$ and $z=0$ and $z=L$; this, or even the validity of (1) between $S$ and $C$ is the Rayleigh hypothesis. ${ }^{29,30}$ A necessary and sufficient condition for this hypothesis to hold is that $u^{(s)}(\mathbf{r})$ may be analytically continued up to the inscribed sphere $C^{\prime}$, i.e., its singularities lie inside that sphere $C^{\prime} .^{29,30}$

Having seen that the scattered field $u^{(s)}(\mathbf{r})$ is an analytic function at least in the exterior domain and providing that $\left|\mathbf{r}_{>}-\mathbf{r}^{\prime}\right| \neq 0,\left(\mathbf{r}^{\prime} \in v\right)$, it remains to see with more detail what sort of analyticity may characterize $u^{(s)}(\mathbf{r})$.

From either Eq. (12) or (13) one can see that

$$
\left|\boldsymbol{A}^{( \pm)}(\mathbf{K})\right|<\boldsymbol{M} \gamma^{\perp^{\prime}}(\mathbf{K})
$$

for some constant $M$, where

$$
\begin{aligned}
& \gamma^{(+)}(\mathbf{K})=e^{+|q| L}, \\
& \gamma^{(-)}(\mathbf{K})=1 .
\end{aligned}
$$

Thus, at points $\mathbf{r}_{>}$where the angular spectrum representation Eq. (11), is valid, one has that the spatial frequency spec$\operatorname{trum} A^{( \pm)}(\mathbf{K}) e^{ \pm \mathrm{iqz} z}$ is bounded by

$$
\left|A^{(+)}(\mathbf{K}) e^{i q z_{>}}\right|<M e^{-|q|\left(z_{>}-L\right)} \text { for } z_{>} \in R^{(+)},
$$




$$
\left|A^{(-)}(\mathbf{K}) e^{-i q z_{>}}\right|<M e^{-|q| z_{>}} \text {for } z_{>} \in R^{(-)} \text {. }
$$

For optical frequencies, given a certain level of noise, and at a distance $z$ large enough it would be impossible to distinguish from zero the bound given by Eqs. (31) for

$$
|\mathbf{K}| \geqslant N k_{0}, \quad(N \geqslant 1)
$$

so that under those conditions the spectrum $A^{(+)}(\mathbf{K}) e^{ \pm i q z_{>}}$is effectively bandlimited to a finite range given by Eq. (32) (see also Ref. 18$)$. Thus, in practice, $u\left(\mathbf{r}_{>}\right)$may be approximately characterized by a function to which the Plancherel-Polya theorem ${ }^{23}$ may be applied for constant $z_{>}$, i.e., the function $u\left(\mathbf{r}_{>}\right)$may be considered as the limit for real values $x_{>}, y_{>}$of an entire function of exponential type in the space of complex variables $\hat{x}_{>}=x_{>}+i x^{\prime}, \hat{z}_{>}=z_{>}+i z^{\prime}>$. [When $u\left(\mathbf{r}_{>}\right)$ is source-free then $A^{\left( \pm{ }^{\prime}\right.}(\mathbf{K})=0$ for $|\mathbf{K}|>k_{0}$ (Ref. 28) and the above will also hold]. This is, of course, an approximation taking into account the presence of noise and at $z_{>} \in\left(R^{(+)}, R^{(-)}\right)$large enough to record the frequency spectrum as of effective finite support. (Of course in an ideal, noise-free situation, the bounds (31) will have a certain value, however small, and the above characterization could not be made.)

This scheme is particularly valuable in situations of cylindrical symmetry along the $O Y$ axis. Then the scattered field $u^{(s)}\left(\mathbf{r}_{>}\right)=u^{(s)}\left(x_{>}, z_{>}\right)$may be extended as an entire function of exponential type into the complex plane of the single complex variable $\hat{x}_{>}=x_{>}+i x_{>}^{\prime}$ at each $z_{>}$constant. As it has been already seen, the information of these functions of one complex variable (in contrast with those of several complex variables) is encoded by the position of its zeros $\hat{x}_{n}$ in the $\hat{x}_{>}$-complex plane by means of the Hadamard product, Eq. (22). These zeros lie asymptotically on a line parallel to the real axis (see Refs. 4 and 5 for a detailed discussion of information encoding by zeros), with a spacing given by the $\mathrm{Ny}-$ quist rate, or Shannon points ${ }^{19}: x_{n}=n \pi / N k_{0}$ and enables one to define a zero density ${ }^{4,5}$ :

$$
\mathscr{D}=\lim _{r \rightarrow \infty} \frac{n(r)}{r}=\frac{N k_{0}}{\pi}=\frac{2 N}{\lambda}, \quad r=\left|\hat{x}_{>}\right| .
$$

As the distance $z_{>}$varies, the configuration of the zeros $\hat{x}_{n}$ at each complex plane $\hat{x}_{>}$associated to the corresponding value of $z_{>}$changes. Therefore, as the scattered field propagates in the exterior domain, the information conveyed by these zeros propagates along "zero trajectories" in a fourdimensional complex space (Fig. 2). This yields another interpretation to Gabor's treatment of information ${ }^{32}$ : each zero encodes a degree of freedom or logon of the scattered wavefunction. The propagation of information along zero trajectories may be associated to the concept of tubes of information. ${ }^{32}$

As $z$, increases however, the effective support of $A^{{ }^{1}{ }^{\prime}(\mathbf{K}) e^{ \pm i q z}>}$ marked by Eqs. (31) decreases until there is no contribution of evanescent waves. This implies that as the scattered field propagates, the zero configuration at each

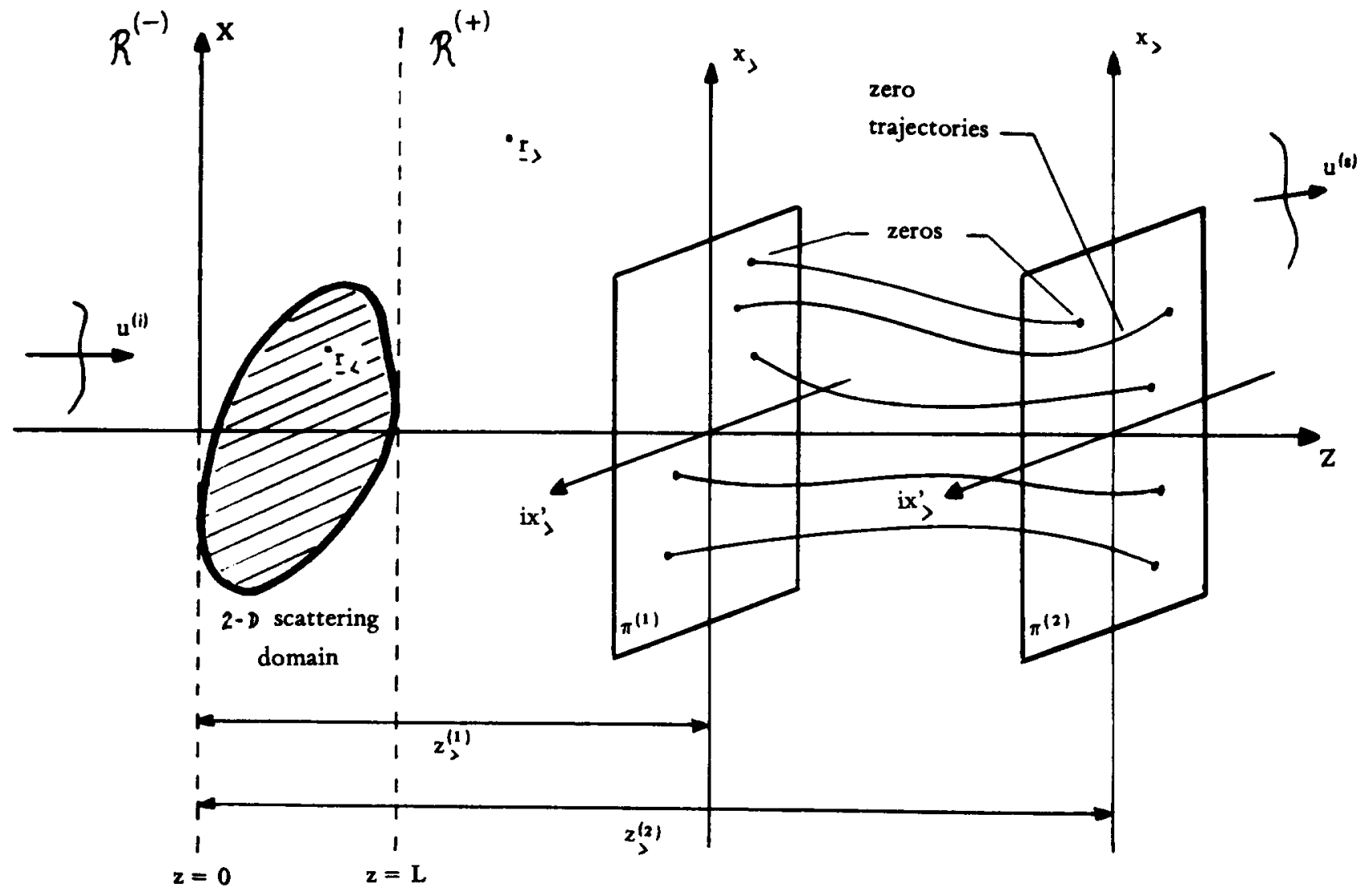

FIG. 2. Propagation of zeros of the scattered field along zero trajectories from the configuration in the $\hat{x}_{>}$-complex plane $\pi^{(1)}$ associated to the distance $z_{>}^{\prime \prime}$ to the positions in the $\hat{x}_{>}$-complex plane $\pi^{(2)}$ associated to the distance $z_{>}^{(2)}>z_{>}^{(1)}$. 
complex plane associated to the corresponding $z$ not only varies, but also the zero density decreases from the value $2 N /$ $\lambda$ until a certain $z_{>}^{(c)}$ at which one reaches the limit marked by the evanescent waves

$$
\mathscr{D}_{0}=\frac{2}{\lambda},
$$

and from that value of $z_{>}^{(c)}$ onwards the density remains constantly equal to $\mathscr{D}_{0}$ as $z_{>}$increases.

This impossibility of recovering higher zero density in the reversal process shows the irreversible nature of information loss as the scattered field propagates. From a zero configuration of density $\mathscr{D}_{0}=2 / \lambda$ in a complex plane $\hat{x}_{>}$associated with a certain distance $z$, one can reconstruct the zero configurations at all prior complex planes (i.e., at smaller $z_{>}$I with the same density $\mathscr{D}_{0}=2 / \lambda$; however, from a well known theorem of the theory of entire functions ${ }^{33}$ (Carlson's theorem), no entire function can have a density of zeros larger than $T / \pi$, if $T$ is its bandwidth, unless it is identically zero. Thus, no entire function can have a zero density larger than $\mathscr{D}_{0}=2 / \lambda$ if evanescent waves are excluded. This fact was already noted by Schmidt-Weinmar ${ }^{21}$ who quoted the impossibility of obtaining information about the field near the sources from the far field containing only homogeneous waves.

Thus, as one considers the situation in which the conjugate wave reproduces in the reversal process the field propagation; i.e., as $z$ tends to a plane $z=z_{0}$, which is the nearest plane to the center of the scattering volume, at which the angular spectrum representation (11) converges, the computation of $\boldsymbol{u}^{(s)}\left(\hat{x}_{>}, z_{>}\right)$at $z_{>}>z_{>}^{(c)}>z_{0}$ will yield only a replica of $u^{(s)}\left(\hat{x}_{0}, z_{0}\right)$ with lower resolution limited to $\lambda / 2$, i.e., with smaller zero density $\mathscr{D}_{0}=2 / \lambda$. This is in agreement with the lack of convergence as a simple limit of $u^{(s)}\left(\hat{x}_{>}, z\right)$ to $u^{(s)}\left(\hat{x}_{0}, z_{0}\right)$, but as a limit in the mean, put forward by Sherman. ${ }^{28}$ Only when $u^{(s)}\left(\hat{x}_{0}, z_{0}\right)$ also has a zero density $\mathscr{D}_{0}=2 /$ $\lambda$, the convergence will be in the form of an ordinary limit [in particular, when $u^{(s)}(\mathbf{r})$ is source-free ${ }^{28}$, since then all the zeros of $u^{(s)}\left(\hat{x}_{0}, z_{0}\right)$ may be found by following backwards the zero trajectories starting from the zero configuration of $u^{(s)}\left(\hat{x}_{>}, z\right)$

\section{SUMMARY AND CONCLUSIONS}

We have generalized and exploited further some aspects of the analyticity properties of scattered scalar fields.

First, performing the analytic continuation of the 3-D Fourier transform $F$ of the source function, we have seen that a single scattering experiment, or a discrete number of them, yields an angular spectrum which is given by the values of $F$ along a line, or lines, on which it may have a zero since it is the boundary value on the real axes of an entire function of exponential type in a 6-D complex space and, as such, it possesses an infinity of zero lines.

Scattering and diffraction are usually regarded as processes creating evanescent waves that are added to the usual homogeneous components of the scattered field. Changing the $\xi$-path of integration we have seen that scattering and diffraction may be equally envisaged as phenomena in which only inhomogeneous (leaky) wave are produced (a study of these waves at planar interfaces may be found in Ref. 34). These inhomogeneous waves correspond to a certain analytic continuation of the scattering amplitude into the complex space $\widehat{\mathbf{K}}$ avoiding the corresponding branch cuts. [This continuation of $\boldsymbol{A}(\mathbf{K})$ has been already used in order to put forward the equivalence between the solution to the phase problem and the location of the zeros of $A(\widehat{K}) .{ }^{4}$ Also the use of inhomogeneous wave representations has been employed in order to obtain information of subwavelength scattering sources $^{35}$ ].

Let us observe that if the source is planar then $A(\hat{\mathbf{K}})$ is an entire function (see also Wolf in Refs. 2 and 21).

Secondly, the scattered field is described in its free propagation outside the scattering volume by an analytic function of the spatial variables. (On the other hand, the generalized analytic character of this field in 2-D problems in the complex variable $x+i z$ leads to integral theorems such as the Helmholtz-Kirchhoff formula and the extinction theorem $\left.{ }^{36}\right)$. The description of the field propagation based on Huygens principle is ultimately a consequence of the possibility of performing an analytic continuation of the wave function outside the scattering volume. At optical frequencies, when evanescent waves from a certain spatial frequency onwards may be neglected given a certain level of noise, this analyticity is more specific: the field may be approximated by an entire function of exponential type. In two-dimensional problems, at each distance $z$, from the object the scattered wave function is therefore described by its zeros in the $\hat{x}_{>}$-plane having a certain density $2 N / \lambda(N \geqslant 1)$. As $z_{>}$increases this density decreases until one reaches the region at which the evanescent waves may be neglected. This yields a density of zeros $2 / \lambda$ and establishes the impossibility of fully reconstructing the field with higher zero densities at smaller distances from the object in the reversal process; and also poses the resolution limit $\lambda / 2$ for the reconstruction of object details (subwavelength source reconstruction requires therefore the localization of zeros with densities above $2 / \lambda$ ). Only in the case of source-free fields, for which the density keeps constantly equal to $2 / \lambda$ in propagation, this reconstruction by means of the inverse process is straightforward.

\section{ACKNOWLEDGMENTS}

The author wishes to acknowledge many interesting discussions with Drs. G. Ross and M. A. Fiddy of Queen Elizabeth College, London, that motivated this work.

He also wants to thank a referee for many valuable comments.

'D. L. Colton, Analytic Theory of Partial Differential Equations (Pitman, London, 1980).

${ }^{2}$ K. Miyamoto and E. Wolf, J. Opt. Soc. Am. 52, 615-625 (1962); E. Wolf, J. Opt. Soc. Am. 68, 1597-1605 (1978).

${ }^{3}$ G. Ross, M. A. Fiddy, M. Nieto-Vesperinas, and I. Manolitsakis, Opt. Acta 26, 229-238 (1979).

${ }^{4} \mathrm{G}$. Ross, M. A. Fiddy, and M. Nieto-Vesperinas, "The inverse scattering problem in structural determinations," in Inverse Scattering Problems in Optics, edited by H. P. Baltes, Topics in Current Physics, Vol. 20 (SpringerVerlag, Berlin, 1980), Chap. 2, pp. 15-17.

${ }^{5}$ M. A. Fiddy, G. Ross, M. Nieto-Vesperinas, and A. M. J. Huiser, Opt. Acta 29, 23-40 (1982). 
${ }^{6}$ G. Ross, M. A. Fiddy, and H. Moezzi, Opt. Acta 27, 1433-1451 (1980). ${ }^{7}$ J. G. Walker, Opt. Acta 28, 1017-1019 (1981); Opt. Acta 28, 735-738 (1981).

${ }^{8} J$. W. Wood, M. A. Fiddy, and R. E. Burge, Opt. Lett. 514-516 (1982).

${ }^{9}$ M. A. Fiddy and G. Ross, Opt. Acta 26, 1139-1146 (1979).

${ }^{10}$ Y. M. Bruck and L. G. Sodin, Opt. Commun. 30, 304-308 (1979).

${ }^{11}$ A. M. J. Huiser and P. Van Toorn, Opt. Lett. 5, 499-501 (1980).

${ }^{12}$ I. Manolitsakis, J. Math. Phys. 23, 2291-2298 (1982).

${ }^{13}$ N. Bleistein and J. K. Cohen, J. Math. Phys. 18, 194-201 (1977).

${ }^{14}$ A. J. Devaney, J. Math. Phys. 19, 1526-1531 (1978).

${ }^{15}$ E. Wolf, Opt. Comm. 1, 153-156(1969); A. Baños, Dipole Radiation in the Presence of a Conducting Half-space (Pergamon, New York, 1966), Eq. (219).

${ }^{16}$ D. N. Pattanayak and E. Wolf, Phys. Rev. D 13, 913-923 (1976).

${ }^{17}$ As is well known, Eq. (1) represents the leading term of the asymptotic expression of (11) for $k_{0} r \rightarrow \infty .^{2}$ As such, the argument $k_{0} s$ in Eq. (1) is restricted to be $\left|k_{0} s_{\perp}\right| \leqslant\left|k_{0} s\right|=k_{0}$ at difference with the vector $\mathbf{K}$ defined in Eq. (11) that can take any real value.

${ }^{18}$ J. R. Shewell and E. Wolf, J. Opt. Soc. Am. 58, 1596-1603 (1968).

${ }^{19} \mathrm{~J}$. W. Goodman, Introduction to Fourier Optics (McGraw-Hill, New York, 1968).

${ }^{20}$ W. H. Carter, J. Opt. Soc. Am. 65, 1054-1058 (1975).

${ }^{21}$ H. G. Schmidt-Weinmar, J. Opt. Soc. Am. 65, 1059-1066 (1975).

${ }^{22}$ G. S. Agarwal and E. Wolf, J. Opt. Soc. Am. 72, 321-326 (1982).

${ }^{23}$ B. A. Fucks, "Introduction to the Theory of Analytic Functions of Several Complex Variables," Trans. Math. Monographs, Vol. 8 (Amer. Math. Soc., Providence, RI, 1983).

${ }^{24}$ A. T. Friberg and E. Wolf, J. Opt. Soc. Am. 73, 26-32 (1983).

${ }^{25}$ W. F. Osgood, Lehrbuch der Funktionentheorie (Teubner, Leipzig, 1929), Vol. 2 .
${ }^{26}$ R. Courant and D. Hilbert, Methods of Mathematical Physics (Interscience, New York, 1962), Vol. 2.

${ }^{27}$ P. R. Garabedian, Partial Differential Equations (Wiley, New York, 1964).

${ }^{28}$ G. C. Sherman, Phys. Rev. Lett. 21, 761-764 (1968), J. Opt. Soc. Am. 59, 697-711 (1969).

${ }^{29}$ R. F. Millar, Proc. Camb. Phil. Soc. 65, 773-791 (1969).

${ }^{30}$ R. F. Millar, Radio Sci. 8, 785-796 (1973); A. J. Devaney and G. C. Sherman, SIAM Rev. 15, 765-786 (1973).

${ }^{31} \mathrm{~A}$ result that may be obtained from the Mie scattering is that the scattered field is analytic up to the origin of a perfectly conducting sphere. Although the analytic continuation of the field inside the sphere differs from the physical field which is actually zero, [V. H. Weston and J. J. Bowman, Ergun Ar, Arch. Rational Mech. Anal. 31, 199-213 (1968); B. J.

Hoenders, "The Uniqueness of Inverse Problems," in Inverse Source Problems in Optics, edited by H. P. Baltes, Chap. 3, pp.41-82, Topics in Current Physics, Vol. 8 (Springer-Verlag, Berlin, 1978)]. I recognize a referee for having drawn attention to this point.

${ }^{32}$ D. Gabor, "Light and information," in Proceedings of the Symposium on Astronomical Optics and Related Subjects, edited by Z. Kopal (NorthHolland, Amsterdam, 1956), pp. 17-30.

${ }^{33}$ R. P. Boas, Entire Functions (Academic, New York, 1954).

${ }^{34}$ T. Tamir, Optik 38, 269-297 (1973).

${ }^{35}$ H. G. Schmidt-Weinmar, "Spatial Resolution of Subwavelength Sources from Optical-Far-Zone Data," in Inverse Source Problems in Optics, edited by H. P. Baltes, Chap. 4, Topics in Current Physics, Vol. 9 (SpringerVerlag, Berlin, 1978).

${ }^{36} \mathrm{M}$. Nieto-Vesperinas, "On the representation of two-dimensional scattered fields in the complex plane," J. Math. Phys. 25, 1592 (1984). 